\title{
Quantification of transmission of foot-and-mouth disease virus caused by an environment contaminated with secretions and excretions from infected calves
}

\author{
Carla Bravo de Rueda ${ }^{1,2}$, Mart CM de Jong ${ }^{2 *}$, Phaedra L Eblé ${ }^{1}$ and Aldo Dekker ${ }^{1}$
}

\begin{abstract}
Foot-and-mouth disease virus (FMDV) infected animals can contaminate the environment with their secretions and excretions. To quantify the contribution of a contaminated environment to the transmission of FMDV, this study used calves that were not vaccinated and calves that were vaccinated 1 week prior to inoculation with the virus in direct and indirect contact experiments. In direct contact experiments, contact calves were exposed to inoculated calves in the same room. In indirect contact experiments, contact calves were housed in rooms that previously had held inoculated calves for three days (either from 0 to 3 or from 3 to 6 days post inoculation). Secretions and excretions from all calves were tested for the presence of FMDV by virus isolation; the results were used to quantify FMDV transmission. This was done using a generalized linear model based on a 2 route ( $2 R$, i.e. direct contact and environment) SIR model that included information on FMDV survival in the environment. The study shows that roughly $44 \%$ of transmission occurs via the environment, as indicated by the reproduction ratio $\hat{R}_{0}^{2 R}$ environment that equalled 2.0, whereas the sum of $\hat{R}_{0}{ }^{2 R}$ contact and $\hat{R}_{0}{ }^{2 R}$ environment equalled 4.6. Because vaccination 1 week prior to inoculation of the calves conferred protective immunity against FMDV infection, no transmission rate parameters could be estimated from the experiments with vaccinated calves. We conclude that a contaminated environment contributes considerably to the transmission of FMDV therefore that hygiene measures can play a crucial role in FMD control.
\end{abstract}

\section{Introduction}

Foot-and-mouth disease virus (FMDV) is the causative agent of foot-and-mouth disease (FMD), a highly contagious disease of livestock. Outbreaks of FMD cause vast sums of money to be spent, to reduce its incidence to low levels [1]. Control measures to restrict the spread of FMDV include movement restrictions, but even when movement restrictions are applied, these do not always prevent new outbreaks (for example in the 2001 FMD epidemic in United Kingdom [2]). Since these restrictions mean that livestock are not allowed to move between farms, direct contact cannot be the (major) cause of transmission, so other, indirect, routes must play a role.

\footnotetext{
* Correspondence: mart.dejong@wur.nl

${ }^{2}$ Department Quantitative Veterinary Epidemiology, Wageningen University, P.O. Box 338, 6700 AH Wageningen, The Netherlands

Full list of author information is available at the end of the article
}

Because most of the secretions and excretions of FMDV infected animals contain virus [3], environmental contamination with secretions and excretions containing FMDV was considered to be one of the causes of FMDV spread [4]. This conclusion was supported by the fact that FMDV remains in the environment, for at least $24 \mathrm{~h}$, after infected animals are killed [5]. Moreover, as studies on survival of FMDV in secretions and excretions have shown, detectable amounts of FMDV persist in the environment (for example, in manure) for up to 14 weeks due to the thermal stability of the virus [6,7]. The suspicion that an environment contaminated with secretions and excretions from FMDV infected animals contributes to the transmission of FMDV has likewise persisted.

SIR (susceptible-infected-recovered) models have been used to model the role of the environment in the transmission of different pathogens [8-12]. Although 
transmission of FMDV has been quantified in animal experiments $[13,14]$ using a stochastic SIR model [15] and a transient-state algorithm [16], such studies have neither modelled nor quantified the contribution of the environment. In addition, FMDV transmission is known to be reduced through vaccination [17], and that vaccinating 2 weeks before inoculation with the virus reduces the reproduction ratio $R_{0}$ to a value below 1 [18]. However, it is unknown whether this could be accomplished through earlier vaccination.

Thus, the aim of the present study is twofold: to utilize a 2 route-SIR model i.e. with both direct contact and indirect (environment) routes, to quantify the contribution of a contaminated environment to the transmission of FMDV, and to examine whether vaccination one week before inoculation with the virus could reduce FMDV transmission through either direct contact or via the environment. As this article shows, a contaminated environment contributes considerably to the transmission of FMDV, and vaccination of cattle 1 week prior to inoculation with the virus does confer protective immunity against FMDV infection.

\section{Materials and methods}

\section{Experimental design}

We used 46 female calves, aged between 6 and 7 months, born and raised in The Netherlands on conventional dairy farms. Our experiments were performed in rooms approximately $10 \mathrm{~m}^{2}$ inside the biosecurity facilities of the Central Veterinary Institute (CVI, Lelystad, The Netherlands). The settings for temperature and humidity in the stables were $20-24{ }^{\circ} \mathrm{C}$ and $40-70 \%$ relative humidity respectively. The experiments received ethical approval from the animal experiment committee of the CVI in accordance with Dutch law. The experiments with nonvaccinated calves and the experiments with vaccinated calves were performed sequentially. During the experiments, all calves were inspected daily for clinical signs of FMD. In these inspections, rectal temperature above $39.5{ }^{\circ} \mathrm{C}$ was considered fever [19] and the calves were checked for the presence of FMD lesions i.e. vesicles. During inspection and/or sampling, animal caretakers changed coveralls and gloves between animal rooms. The animal rooms in which the indirect transmission experiments were performed were not cleaned with water; instead, animal waste was swept daily with a broom to the drainage.

\section{Challenge virus and vaccine}

Virus inoculation was performed intranasally using FMDV Asia-1 TUR/11/2000. The inoculum contained $10^{6.1}$ plaque forming units $(\mathrm{pfu}) / \mathrm{mL}$ (titrated on primary lamb kidney cells). Each inoculated calf received $1.5 \mathrm{~mL}$ of inoculum per nostril. The vaccine used was a freshly prepared inactivated
FMDV Asia-1 Shamir vaccine, prepared in a double water-in-oil emulsion. The potency of a similarly prepared vaccine was previously determined at $>6 \mathrm{PD}_{50}$ (at 28 days post vaccination).

\section{Direct contact experiments}

In both vaccinated and unvaccinated scenarios, 10 calves were randomly assigned to 5 animal rooms in pairs i.e. 2 calves per room. On the day of inoculation i.e. 0 days post inoculation (dpi), 1 calf from each pair was moved to a separate animal room and inoculated with FMDV. Eight hours after inoculation, these calves were reunited with their original roommates. In the experiment in which vaccinated calves were used, all 10 calves were vaccinated intramuscularly with $2 \mathrm{~mL}$ of vaccine one week before inoculation $(-7 \mathrm{dpi})$. The direct contact experiments ended at $14 \mathrm{dpi}$, assuming this duration could allow transmission to occur.

\section{Indirect contact experiments}

This experimental design is shown in Figure 1. In both vaccinated and unvaccinated scenarios, 4 calves were inoculated with FMDV at 0 dpi (2 pairs (groups A and B) of inoculated calves, IA and IB). Eight hours after inoculation, they were moved into 2 animal rooms to which they had been randomly assigned, 2 calves per room. At $3 \mathrm{dpi}$, the inoculated calves were moved to 2 new animal rooms. Subsequently, 1 pair of non-vaccinated contact calves (contacts 1, C1A and C1B) was moved into each of the animal rooms that had been contaminated by the inoculated calves. The inoculated calves stayed in their new rooms from 3 to $6 \mathrm{dpi}$; at $6 \mathrm{dpi}$, they were removed from the animal rooms and euthanized. On the same day, each of these now-contaminated rooms was allocated to a pair of non-vaccinated contact calves (contacts $2, \mathrm{C} 2 \mathrm{~A}$ and $\mathrm{C} 2 \mathrm{~B})$. In the experiment in which vaccinated calves were used, at -7 dpi the 4 inoculated calves were vaccinated intramuscularly with $2 \mathrm{~mL}$ of vaccine. The 8 contact calves were not vaccinated. The indirect contact experiments ended at $20 \mathrm{dpi}$.

\section{Vaccine controls}

During the experiment with vaccinated calves, 2 additional calves were vaccinated and used as vaccine control group to evaluate the serological response of the calves in the absence of infection; these controls were housed together in a separate animal room.

\section{Sampling}

Oropharyngeal fluid (OPF) swabs, heparinised blood, urine and faeces samples were collected daily from each calf from $0 \mathrm{dpi}$ until the end of the experiment. OPF was collected by inserting a cotton gauze with a $25 \mathrm{~cm}$ long forceps into the mouth of the calves and by rubbing 


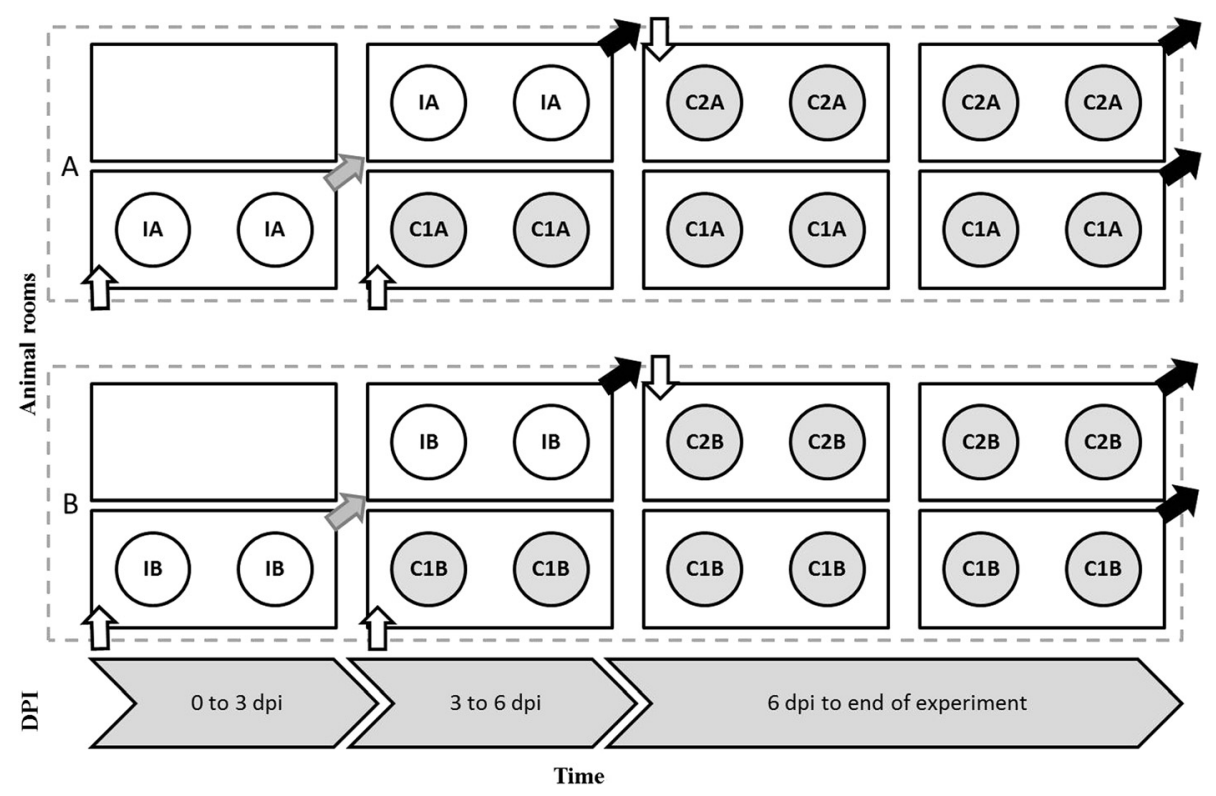

Figure 1 Indirect contact experiment design. Panels $\mathbf{A}$ and $\mathbf{B}$ represent groups A and B. IA and IB, calves inoculated at 0 days post infection (dpi); C1A and C1B, contact exposed calves to contaminated environment from 0 to 3 dpi; $C 2 A$ and C2B, contact exposed calves to contaminated environment from 3 to 6 dpi. Grey arrows indicate movement of animals to an (- other) animal room. Black arrows indicate movement of animals for euthanasia.

the surface of the oropharyngeal cavity. In the laboratory, the pieces of cotton gauze were immersed in $4 \mathrm{~mL}$ of Eagle's minimum essential medium (EMEM) containing $2 \%$ fetal calf serum (FCS) and $10 \%$ antibiotics solution (ABII: $1000 \mathrm{U} / \mathrm{mL}$ of penicillin, $1 \mathrm{mg} / \mathrm{mL}$ of streptomycin, $20 \mu \mathrm{g} / \mathrm{mL}$ of amphotericin $\mathrm{B}, 500 \mu \mathrm{g} / \mathrm{mL}$ of polymixin $\mathrm{B}$, and $10 \mathrm{mg} / \mathrm{mL}$ of kanamycin). After $20 \mathrm{~min}$ of incubation at environmental temperature, the samples were centrifuged (2500 rpm for $15 \mathrm{~min}$ ). Samples were stored at $-70{ }^{\circ} \mathrm{C}$ until virus isolation and real-time reverse transcriptase polymerase chain reaction (RT-PCR) analysis.

Heparinised blood samples (10 mL per calf) for virus isolation were taken daily, while clotted blood samples (10 mL per calf) for serology were taken twice per week. Blood samples were centrifuged at $2500 \mathrm{rpm}$ for $15 \mathrm{~min}$. Plasma was stored at $-70{ }^{\circ} \mathrm{C}$ until virus isolation analysis and serum was stored at $-20{ }^{\circ} \mathrm{C}$ until serological analysis. Urine samples were collected, as calves were stimulated to urinate spontaneously by rubbing the skin next to the vulva. Urine samples were collected into sterile plastic containers. In the laboratory, $800 \mu \mathrm{L}$ of urine was mixed with $200 \mu \mathrm{L}$ of a $50 \%$ FCS, $50 \%$ ABII solution and stored at $-70{ }^{\circ} \mathrm{C}$ until virus isolation analysis.

Faeces samples were collected from the rectum. In the laboratory, the faeces was suspended 1:10 (w/v) in EMEM containing 10\% FCS and 10\% ABII solution, and vortexed with glass beads. After 20 min of incubation at environmental temperature, the suspension was vortexed and centrifuged (3000 rpm for $15 \mathrm{~min}$ ). The supernatants were stored at $-70{ }^{\circ} \mathrm{C}$ until virus isolation analysis.

\section{Virus detection}

All OPF, heparinised blood, urine and faeces suspension samples were tested for presence of FMDV by plaque count on monolayers of secondary lamb kidney cells (virus isolation, VI). Samples were tested in 2 wells of a six-well plate using $200 \mu \mathrm{L}$ per well, as previously described [20]. All OPF samples were also tested for presence of FMDV by RT-PCR. RNA isolation was performed using the Magna Pure LC total Nucleid Acid Isolation kit (Roche) and the MagNa Pure 96 system $^{\circ}$ (Roche). Isolated RNA was tested in a LightCycler 480 Real-Time PCR System $^{\circ}$ (Roche) using a QuantiFast Probe RT-PCR kit ${ }^{\circ}$ (Qiagen), all in accordance with the manufacturers' instructions. The primers, probes and test protocol used have been previously described [21].

\section{Statistical analysis of virus secretions and excretions}

Using data from both the direct and the indirect contact experiments, we calculated, for individual animals, the area under the curve (AUC) of the virus titres. The AUC represents the total amount of FMDV that was secreted and excreted by the infected calves during the experiment. The AUCs were calculated for each calf using the non-logarithm-transformed virus titres observed in its OPF swabs, urine and faeces samples. In the statistical analysis, the logarithm of the AUC was used (log AUC). The maximum FMDV log titres found in OPF swabs, urine and faeces samples from each calf were also calculated. The duration (in days) of FMDV secretion and excretion in OPF swabs, urine and faeces samples was calculated for 
each calf, counting from the first day until the last day the calf tested positive in the virus isolation assay (in either OPF swabs, urine or faeces samples). A Kruskal Wallis test was used to test whether differences existed between the experimental groups (i.e. inoculated calves, direct contacts, indirect contacts $\mathrm{C} 1$ and indirect contacts $\mathrm{C} 2$ ) for either the $\log \mathrm{AUC}$, the maximum FMDV log titres or the duration of FMDV secretion and excretion. The log AUC and the maximum FMDV log titres were tested for each type of sample (OPF swabs, urine and faeces). The duration of FMDV secretion and excretion was tested using data from OPF swabs, urine and faeces samples combined.

\section{Antibody detection}

A commercially available ELISA (PrioCHECK ${ }^{\circ}$ FMDV NS, Prionics) was used to detect antibodies against nonstructural proteins of FMDV. The test was performed in accordance to the manufacturer's instructions. This test detects antibodies against the non-structural protein 3B of FMDV and differentiates infected from non-infected animals in both non-vaccinated and vaccinated animals. Samples were considered to be positive when the percentage of inhibition was $\geq 50 \%$. The virus neutralization test (VNT) was performed as previously described [22] but using BHK-21 cells instead of porcine kidney cells. Titres were determined against both the vaccine strain (Asia-1 Shamir) and the challenge strain (Asia-1 TUR/11/2000). Samples were considered to be positive when the titres were above $1.2{ }^{10} \log$ (cut-off of validated diagnostic test) using the Asia-1 Shamir strain and $0.6{ }^{10} \log$ (cut-off based on the score of control samples) using the Asia-1 TUR/11/2000 strain.

\section{Quantification of the FMDV survival rate}

The FMDV survival rate $\left(\sigma\right.$ day $\left.^{-1}\right)$, needed for the calculation of the contribution of the environment $\left(\mathrm{E}_{t}\right)$ to the transmission of FMDV, was calculated using published data on FMDV thermal inactivation combined with own laboratory data. Because the temperature in the animal rooms was approximately $20{ }^{\circ} \mathrm{C}$ during the experiments, the survival rate $\sigma$ was estimated at $20^{\circ} \mathrm{C}$. The lowest, middle and highest estimates of the time needed for a 10-fold reduction in FMDV titres at $20{ }^{\circ} \mathrm{C}$ was used to calculate the FMDV survival rate $\sigma$. An additional file shows the calculation of the FMDV survival rate $\sigma$ in more detail (Additional file 1 with references [7,20,23-27]).

\section{Quantification of FMDV transmission}

Transmission rate parameters: $\beta, \beta_{\text {contact }}$ and $\beta_{\text {environment }}$

The transmission rate parameter $\beta$ is defined as the average number of new infections caused by one typical infectious individual per day in a totally "susceptible" (not infected) population [16,28] (Additional file 2: equations 1 and 2, with references $[16,28,29])$. For the analysis, as described previously [28], it was assumed that the calves were infectious (I) when one of their samples (OPF swabs, urine or faeces) tested positive in the virus isolation assay at the start of the time interval. Contact animals were considered cases (C) when one of their samples (OPF swabs, urine or faeces) tested positive, for the first time, in the virus isolation assay at the end of the time interval. The number of new cases (C) during that time interval is binomially distributed with probability $p$ (which is a function of the transmission rate parameter $\beta$, the number of infected animals $\left(I_{t}\right)$ and the total number of animals $(\mathrm{N})$ ) and with binomial total $\mathrm{S}_{\mathrm{t}}$, the number of susceptible animals. Thus, the probability of a single susceptible animal becoming infected during a period $\Delta \mathrm{t}$ is, $p=1-e^{-e^{C_{0}} \times \frac{I_{t}}{N_{t}} \times \Delta t}$, where $e^{C_{0}}$ is the transmission rate parameter $\beta$. To quantify $\beta$, the data from the direct contact experiment were analysed using a generalized linear model (GLM) [30]. The GLM is based on the binomial distribution and the above-mentioned expression for $p$, using a complementary log-log link function, $\mathrm{S}$ as binomial total, a binomial error function and with $\log \left(\frac{I_{t}}{N_{t}} \times \Delta t\right)$ as offset $[16,28]$. This model will be hereinafter referred to as the 1 route-SIR (1R-SIR) model. To quantify the contribution of the environment to the transmission of FMDV, as an extra route to the 1R-SIR model (Figure 2), we included the environment (E). In the new 2 route-SIR model (2R-SIR) we additionally assumed that the amount of FMDV present

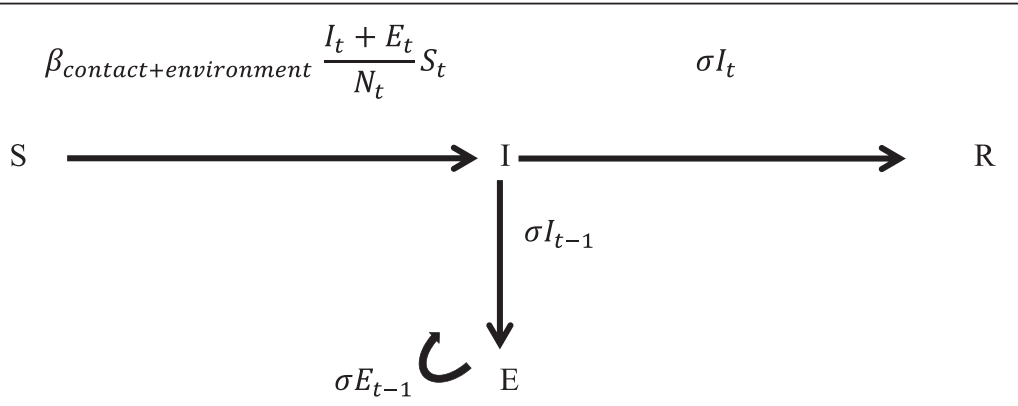

Figure 2 The $\mathbf{2 R}$ - SIR model. The combined transmission rate parameter ( $\beta_{\text {contact+environment }}$ ) depends on the number of infectious calves $\left(I_{t}\right)$ and/or on the amount of virus in the environment $\left(E_{t}\right)$. $E_{t}$ depends on FMDV secretion and excretion by the infected calves on previous days (t-1) and on the remaining amount of FMDV in the environment weighted by $\sigma$. 
in the environment on a specific day $\left(\mathrm{E}_{\mathrm{t}}\right)$ depends on the secretion and excretion of FMDV by infectious individuals (either I or C) on the previous days, as well as on the remaining FMDV in the environment $\left(\mathrm{E}_{(\mathrm{t}-1)}\right)$, both weighted (discounted) by the FMDV survival rate $(\sigma) . E_{t}$ is represented by the following equation: $E_{t}=\sigma I_{(t-1)}+\sigma C_{(t-1) \rightarrow}$ ${ }_{t}+\sigma E_{(t-1)}$ with starting condition $\mathrm{E}_{0}=0$ (Additional file 2: equation 3). We performed a sensitivity analysis in which we multiplied the new cases $(C)$ in the equation above either by 0 or by 0.5 , instead of 1 as it is in the above equation for $E_{t}$, to check whether this affected the outcome. Additionally, we performed a sensitivity analysis in which we considered a latent period (counting the inoculated calves as infected but not yet infectious, (1,2 and 3 days before virus shedding was detected), to check whether the use of an SEIR (susceptible-exposed-infected-recovered) instead of an SIR model would lead to different results for the estimated $\beta$ and $R$ values (i.e. if $\beta$ is underestimated) and whether this affected the estimation of the environmental component.

In the 2R-SIR model, there are 2 ways by which the susceptible calves $\left(S_{t}\right)$ can become infected: (1) because they have been in direct contact with an infectious calf $\left(\mathrm{I}_{\mathrm{t}}\right)$ i.e. being in the same room at the same day as an infectious calf and/or (2) because they have been in contact with a contaminated environment $\left(E_{t}\right)$ i.e. being in an animal room that housed previously one or more infectious individuals (Figure 2). By using the 2R-SIR model, we quantified the transmission rate parameters $\beta_{\text {contact }}$ and $\beta_{\text {environment. }}$ As in the definition of $\beta$ the transmission rate parameter $\beta_{\text {contact }}$ is defined as the average number of new infections per day caused by direct contact to one typical infectious individual in a fully susceptible population. The transmission rate parameter $\beta_{\text {environment }}$ is defined as the average number of new infections per day caused by virus in the environment, where the unit of infectivity is equal to the amount of virus secreted and excreted during one day by an infectious animal. An additional file shows the 2R-SIR model in more detail (Additional file 2: equations 4 to 6 ). In the 2R-SIR model, the number of new cases $\left(C_{t \rightarrow(t+1)}\right)$, whether caused by $I_{t}$ and/or $E_{t}$, is binomially distributed with parameter $p$ as before (see also below) but now $\beta=e^{C_{0}+C_{1} \times f e}$ where $\mathrm{f}_{\mathrm{e}}=\frac{E_{t}}{I_{t}+E_{t}}$ is the fraction of transmission by the environment and therefore its regression coefficient measures the extra infectivity contributed by the environment. When only direct contact can occur, $f_{e}$ is 0 and thus $\beta_{\text {contact }}=e^{C_{0}}$. When only environmental exposure can occur, $\mathrm{f}_{\mathrm{e}}$ is 1 and $\beta_{\text {environment }}=e^{C_{0}+C_{1}}$ (Additional file 2). The latter expression contains $c_{0}+c_{1}$ and thus $c_{1}$ is the extra transmission for each unit of infectivity through the environment as compared to one unit through direct contact. Thus the probability of a susceptible animal becoming infected during a period $\Delta \mathrm{t}$ is $p=1-e^{-e^{C_{0}+f_{e} \times C_{1}} \times \frac{I_{t}+E_{t}}{N_{t}} \times \Delta t}$ (Additional file 2: equation 6). To quantify $\beta_{\text {contact }}$ and $\beta_{\text {environment }}$ we analysed the combined data from both the direct contact experiment and the indirect contact experiment using a GLM. The GLM was based on the binomial distribution and the above mentioned expression for $p$ using a complementary log-log link function, $\mathrm{S}$ as binomial total, a binomial error function, $f_{e}$ as the explanatory variable [28] and with $\log \left(\frac{I_{t}+E_{t}}{N_{t}} \times \Delta t\right)$ as offset (Additional file 2: equations 7 and 8 ). To test whether $\beta_{\text {contact }}$ and $\beta_{\text {environment }}$ were significantly different from each other, we used the Wald test on the regression coefficient of $\mathrm{f}_{\mathrm{e}}$. Both analyses (of the $1 \mathrm{R}$-SIR and of the 2R-SIR models) were performed using the statistical program $R$ [31] and the package stats.

\section{Infectious periods: $T$ and $\tau$}

The infectious period $\mathrm{T}$ was defined as the average infectious period of the inoculated calves that caused transmission from the direct contact experiment. The infectious period of each inoculated calf was defined as the time between the first and the last day on which FMDV was detected (by virus isolation) in OPF swabs, urine, or faeces samples. The $95 \%$ confidence intervals (CI) of $\hat{\mathrm{T}}$ were calculated using the logarithm of $\mathrm{T}(\log$ $\mathrm{T})$ and the variance of $\log \mathrm{T}$ i.e. $e^{\log T \pm 1.96 \sqrt{\operatorname{var}(\log T)}}$. The infectious period $\tau$ represents the infectious period of the contaminated environment. The calculation of $\tau$ was based on the amount of infectious material present in the environment $\left(E_{t}\right.$, used in the 2R-SIR model). Considering the loss of infectiousness due to inactivation at environmental temperature, $\tau$ was calculated by taking the sum of geometric series: $\tau=\sum_{i=1}^{\infty} \sigma^{i} \hat{T}=\hat{T}\left(\frac{1}{1-\sigma}-1\right)$ where $\sigma$ is the survival rate of FMDV and $\hat{T}$ is the estimated average infectious period of the inoculated calves in the direct contact experiment. The method allowed us to obtain an average period over which one infectious animal contributes to the contamination of the environment, weighted for the amount of infectious material relative to the amount secreted and excreted by an infectious animal on one day. The $95 \% \mathrm{CI}$ of $\hat{\tau}$ was calculated using the $95 \%$ CI of $\hat{T}$.

\section{Reproduction ratio $R_{0}$} Using the $1 R$-SIR model: $R_{0}{ }^{1 R}$

The reproduction ratio $R_{0}{ }^{1 R}$ is defined as the average number of new infections caused by one typical infectious individual in a population made up entirely of susceptible individuals [32]. $R_{0}{ }^{1 R}$ was estimated by multiplying the transmission rate parameter $\hat{\beta}$ by the infectious period $\hat{T}$. The $95 \% \mathrm{CI}$ of $\hat{R}_{0}{ }^{1 R}$ was calculated using the variance and the regression constant of the GLM result $(\log \beta)$ and the 
variance and the logarithm of the average infectious period T, i.e. $e^{\log \beta+\log T \pm 1.96 \sqrt{\operatorname{var}(\log \beta)+\operatorname{var}(\log T)}}$.

\section{Using the 2R-SIR model: $R_{0}{ }^{2 R}$ contact, $R_{0}^{2 R}$ environment and $R_{0}^{2 R}$} The reproduction ratio $R_{0}{ }^{2 R}$ is defined as the average number of new infections caused by both direct contact to one typical infectious individual in a population made up entirely of susceptible individuals and the virus left in the environment by that one typical infectious individual on the previous days. Both $R_{O}{ }^{2 R}$ contact and $R_{O}{ }^{2 R}$ environment were estimated using the results from the $2 \mathrm{R}-\mathrm{SIR}$ model, i.e. estimated transmission rate parameter $\hat{\beta}$ contact and $\hat{\beta}$ environment. The $R_{O}{ }^{2 R}$ contact was estimated by multiplying $\hat{\beta}_{\text {contact }}$ by the infectious period $\hat{T}$. The $R_{0}{ }^{2 R}$ environment was estimated by multiplying $\hat{\beta}_{\text {environment }}$ by the infectious period $\hat{\tau}$. Subsequently $R_{O}^{2 R}$ was estimated by summing $\hat{R}_{0}{ }^{2 R}$ contact and $\hat{R}_{0}{ }^{2 R}$ environment. The $\hat{R}_{0}{ }^{2 R}$ contact is the contribution to $\hat{R}_{0}{ }^{2 R}$ by direct contact to virus from an infectious individual (on the day virus secretion and excretion is detected by virus isolation). The $\hat{R}_{0}{ }^{2 R}$ environment is the contribution to $\hat{R}_{0}{ }^{2 R}$ by the virus left in the environment by infectious individuals on previous days. The 95\% CI of $\hat{R}_{0}{ }^{2 R}$ contact, $\hat{R}_{0}{ }^{2 R}$ environment and of $\hat{R}_{0}{ }^{2 R}$ were calculated. For this purpose, we used the variances and the regression constants (see above $c_{0}$ and $c_{1}$ in equation for $p$ ) of the GLM results ( $\log \beta_{\text {contact }}$ or $\left.\log \beta_{\text {environment }}\right)$ and the variances and the logarithm of the average infectious periods $(\log \mathrm{T}$ or $\log \mathrm{T})$. Thus, the $95 \% \mathrm{CI}$ of the $\hat{R}_{0}{ }^{2 R}$ contact is $e^{\log \beta_{\text {contact }}+\log T \pm 1.96 \sqrt{\operatorname{var}\left(\log \beta_{\operatorname{contact}}\right)+\operatorname{var}(\log T)}}$ and, the $95 \% \quad \mathrm{CI}$ of the $\hat{R}_{0}{ }^{2 R}$ environment is $e^{\log \beta_{\text {environment }}+\log T+\log a \pm 1.96} \sqrt{\operatorname{var}\left(\log \beta_{\text {environment }}\right)+\operatorname{var}(\log T)}$ where a is $\left(\frac{1}{1-\sigma}-1\right)$. As $\hat{R}_{0}{ }^{2 R}$ is the sum of $\hat{R}_{0}{ }^{2 R}$ contact and $\hat{R}_{0}{ }^{2 R}$ environment, its variance is $\operatorname{var}\left(e^{\log \left(\hat{R}_{0}^{2 R}\right)}\right)=\operatorname{var}$ $\left(e^{\log \left(\hat{R}_{0}^{2 R} \text { contact }\right)}\right)+\operatorname{var}\left(e^{\log \left(\hat{R}_{0}^{2 R} \text { environment }\right)}\right)$ and although this is not a linear function we calculated the $95 \%$ CI of the $\hat{R}_{0}{ }^{2 R} \quad$ using: $\operatorname{var}\left(e^{\log \left(\hat{R}_{0}^{2 R}\right)}\right)=e^{\operatorname{var}\left(\log \left(\hat{R}_{0}^{2 R} \text { contact }\right)\right)}$ $+e^{\operatorname{var}\left(\log \left(\hat{R}_{0}^{2 R} \text { environment }\right)\right)}$.

\section{Using the final size model: $R_{0}{ }^{F S}$}

The transmission parameter $R_{O}$ can also be estimated based only on the final outcome (the final size of the experiment, FS) [33]. We estimated the $R_{0}^{F S}$ based on the total number of infected calves at the end of the direct contact experiment under the assumption that the epidemic process ended before the experiment stopped [34]. The animals were considered infected when one or more of their samples tested positive in the virus isolation assay. Because in the direct contact experiment we got all 4 contacts infected in the 4 pairs in which the inoculated calf was considered to be infectious, we used continuity correction, i.e. 3.5 infections in 4 experiments, to avoid an infinite estimate for $R_{0}^{F S}$. The $95 \%$ confidence intervals (CI) of $\hat{R}_{0}{ }^{F S}$ were estimated under the FS assumption by using the binomial distribution for the infected fraction $[33,35]$.

\section{Results}

\section{Experiments with non-vaccinated calves}

Table 1 summarizes the results from the direct and indirect contact experiments with the non-vaccinated calves. FMDV transmission to the contact calves occurred in both experiments.

\section{Direct contact experiment \\ Inoculated calves}

FMD clinical signs were observed in 4 of the 5 inoculated calves. Three of these calves (calves 3643, 3645 and 3649) showed fever and had FMD lesions on the tongue. One of these 3 calves (calf 3643) also had hoof lesions, and another (calf 3651) showed FMD lesions on the nose. Three of the clinically infected calves (calves 3643, 3645 and 3649) shed FMDV in OPF, blood and urine (Table 1). One of these 3 calves (calf 3645) also shed FMDV in faeces. The fourth clinically infected calf (calf 3651) shed FMDV in OPF only. All the inoculated calves were positive in OPF by RT-PCR. Antibodies against non-structural proteins and neutralizing antibodies against FMDV were detected in serum samples from all the inoculated calves. Inoculated calf 3647 became subclinically infected, but shed FMDV in urine, was positive for FMDV in OPF by RT-PCR and developed antibodies against non-structural proteins and neutralizing antibodies against FMDV.

\section{Contact calves}

Clinical signs were observed in the 3 contact calves (calves 3644, 3646 and 3650) that were housed together with inoculated calves 3643, 3645 and 3649. The 3 contact calves showed fever and had FMD lesions on the tongue (calf 3646) and hooves (calves 3644 and 3650); they shed FMDV in OPF, blood and urine (Table 1). One of these 3 calves (calf 3650) also shed FMDV in faeces. Another contact calf (calf 3652) became subclinically infected; it shed FMDV in its OPF. Calves 3644, 3646 and 3650 were positive for FMDV in OPF by RT-PCR. All 4 contact calves in which the virus was detected showed antibodies against non-structural proteins and neutralizing antibodies against FMDV. Calf 3648, in contact with inoculated calf 3647 , showed no FMD clinical signs and tested negative for FMDV and for antibodies against FMDV. Thus transmission occurred in 4 of the 5 animal 
Table 1 Results of virus isolation, RT-PCR (OPF swabs only), antibody detection and detection of FMD clinical signs

Exp $^{\text {a }}$ Calf ID I:C $C^{b}$ Group FMDV detection by virus isolation in OPF swabs (in log10 titres),blood(v), urine(u) and faeces(f) samples and by RT-PCR (OPF

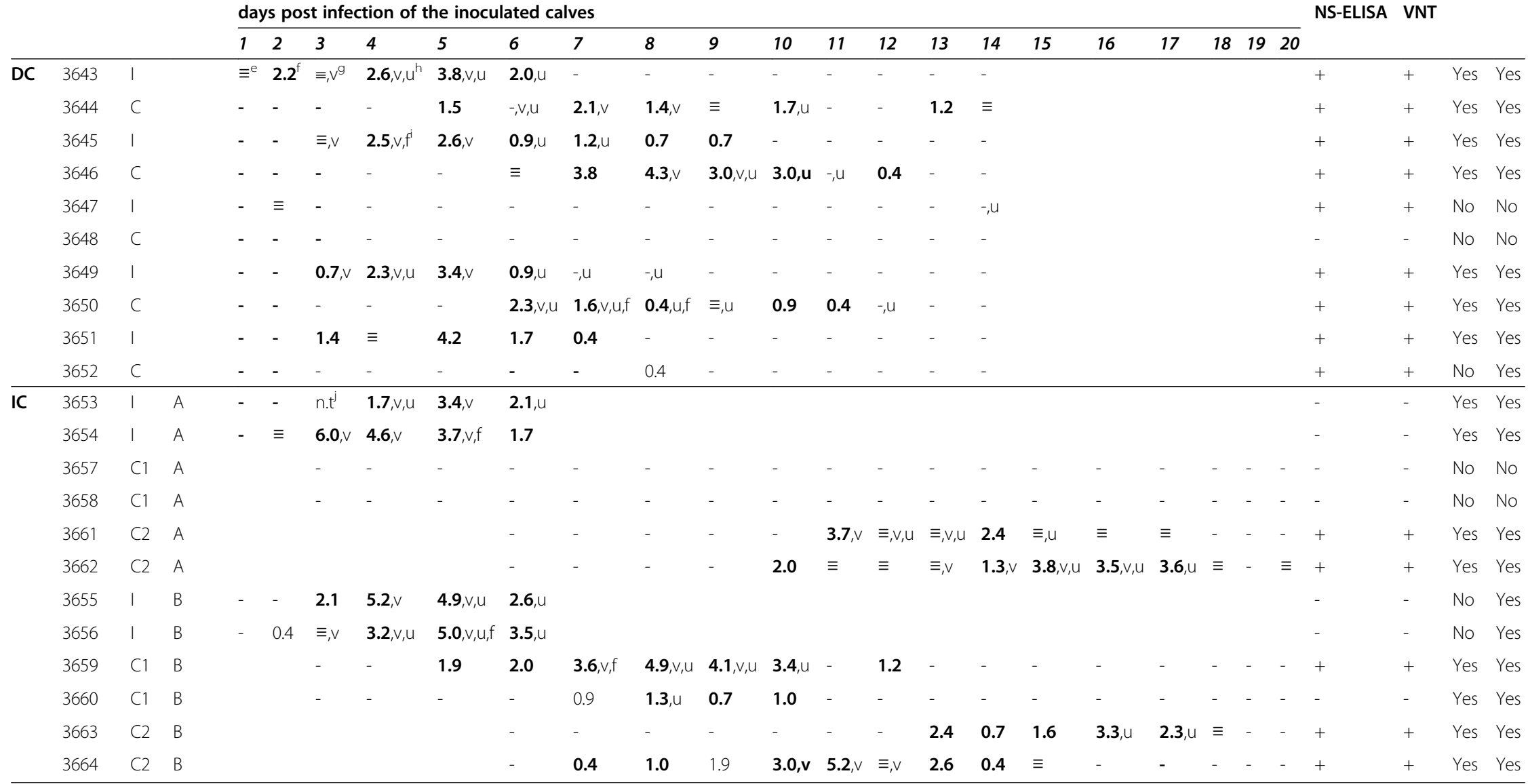

${ }^{\mathrm{a}}$ Exp=experiment: $\mathrm{DC}=$ direct contact, $\mathrm{IC}=$ indirect contact; ${ }^{\mathrm{b}} \mathrm{l}=$ inoculated, $\mathrm{C}=$ contact animal; ${ }^{\mathrm{c} C l i n=c l i n i c a l}$ signs; ${ }^{\mathrm{d}}$ Inf=infectious; ${ }^{\mathrm{e}}$ results of virus isolation (VI) and RT-PCR of oral swab sample: $-=\mathrm{VI}$ and RT-PCR negative, $\equiv=$ VI negative and RT-PCR positive; ${ }^{f}$ oral swab sample scored positive for FMDV by VI (log10 pfu/mL), RT-PCR positive samples are indicated in bold; ${ }^{\mathrm{g}} \mathrm{V}=\mathrm{viraemia}$ : blood sample scored positive for FMDV by Vl;

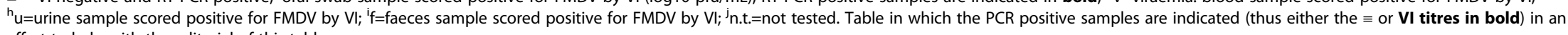
effort to help with the editorial of this table. 
rooms in the direct contact experiment. The only moment infectious virus was recovered from inoculated calf 3647 (from urine) was at $14 \mathrm{dpi}$, at the day of the end of the experiment. Thus, occurrence of transmission was not possible anymore and this pair of calves (calves 3647 and 3648) was excluded from the estimation of the transmission rate parameters and the reproduction ratio.

\section{Indirect contact experiment Inoculated calves}

Clinical signs were observed in 2 out of 4 inoculated calves (number 3653 and 3654; both in pair IA). These 2 inoculated calves showed fever and 1 of them had lesions on the tongue. The other 2 calves (calves 3655 and 3656; pair IB) showed no FMD specific clinical signs. In all 4 inoculated calves, virus was detected in the OPF (IA and IB). All four secreted and excreted FMDV in their blood, urine and/or faeces (Table 1). They all were positive for FMDV in OPF by RT-PCR. Thus, inoculated calves 3655 and 3656 were subclinically infected. Serum samples from all 4 inoculated calves were obtained only at $0 \mathrm{dpi}$ and $3 \mathrm{dpi}$; in these samples neither antibodies against non-structural proteins nor neutralizing antibodies against FMDV were detected as expected.

\section{Contact calves $\mathrm{C} 1$}

Contact calves $\mathrm{C} 1$ were exposed to the animal rooms that were contaminated by the inoculated calves from 0 to 3 dpi. The contact calves of group C1A (calves 3657 and 3658) did not get infected; no FMD specific clinical signs were seen and both calves tested negative by virus isolation, by RT-PCR and, for antibodies against FMDV. The contact calves of group C1B (calves 3659 and 3660) showed fever and one had FMD lesions on the mouth, tongue, nose and hooves. Both $\mathrm{C} 1 \mathrm{~B}$ calves had virus detected in their OPF; one of them secreted and excreted virus in blood, urine and faeces, the other one excreted virus in urine. They tested positive for FMDV in OPF by RT-PCR. One C1B calf showed antibodies against nonstructural proteins in serum (calf 3660). Both C1B calves showed neutralizing antibodies in serum.

\section{Contact calves $\mathrm{C} 2$}

Contact calves $\mathrm{C} 2$ were exposed to the animal rooms that were contaminated by the inoculated calves from 3 to $6 \mathrm{dpi}$. All the contact calves of groups $\mathrm{C} 2 \mathrm{~A}$ and $\mathrm{C} 2 \mathrm{~B}$ showed clinical signs. Three of them showed fever, and all of them showed FMD lesions on the nose and in the mouth. In all 4 calves, virus was detected in their OPF (Table 1); the calves secreted and/or excreted FMDV in the blood (calves 3661, 3662 and 3664) and in urine (calves 3661, 3662 and 3663). They all were positive for FMDV in OPF by RT-PCR. All developed antibodies against non-structural proteins as well as neutralising antibodies. Thus transmission occurred in the indirect contact experiment in 1 of the 2 animal rooms that were contaminated from 0 to $3 \mathrm{dpi}$ and, in both of the animal rooms that were contaminated from 3 to $6 \mathrm{dpi}$.

\section{Statistical analysis of virus secretion and excretion}

The mean values for the AUC's, peak of virus shedding and duration of virus shedding (and their ranges) for OPF swabs, urine samples, faeces samples and blood samples for the inoculated group, the direct contact group and the indirect contact groups $\mathrm{C} 1$ and $\mathrm{C} 2$ are shown in Additional file 3.

No significant difference in log AUC could be determined between the different experimental groups i.e. inoculated, direct contacts, indirect contacts $\mathrm{C} 1$ and indirect contacts $\mathrm{C} 2$, neither for OPF swabs nor for urine nor for faeces $(p>0.05)$. No significant difference in the maximum FMDV log titres was found between the different experimental groups neither for OPF swabs nor for urine nor for faeces $(p>0.05)$. No significant difference in the duration of FMDV secretion and excretion could be determined between the different experimental groups $(p>$ 0.05) (Additional file 3).

\section{Experiments with vaccinated calves}

At day of challenge ( $0 \mathrm{dpi}, 7$ days post vaccination), the average virus neutralisation test (VNT) titre against the vaccine strain FMDV Asia-1 Shamir for all the vaccinated calves (including the vaccine controls) was 2.2

${ }^{10} \mathrm{log}$. The average virus neutralisation test (VNT) titre against the challenge strain FMDV Asia-1 TUR/11/2000 was $1.2{ }^{10} \log$.

\section{Direct contact experiment}

After challenge, neither the vaccinated inoculated calves nor the vaccinated contact calves showed clinical signs of FMD and all calves tested negative by virus isolation and RT-PCR. Only 2 inoculated calves (calves 3972 and 3976) developed antibodies against non-structural proteins.

\section{Indirect contact experiment}

After challenge, neither the vaccinated inoculated calves nor the non-vaccinated contact calves showed clinical signs of FMD. All calves tested negative by virus isolation and RT-PCR. Neither the vaccinated inoculated nor the non-vaccinated contact calves showed detectable antibodies against non-structural protein.

\section{FMDV survival rate $(\sigma)$}

From the combined published and own experimental data, it was estimated that at $20{ }^{\circ} \mathrm{C}$ a 10 -fold reduction in FMDV titres occurs in 2.4 days (95\% CI: 1.7, 3.3). We calculated the FMDV survival rate $(\sigma)$ using the lowest (in spiked urine), middle (in spiked faeces) and highest (in 
spiked buffered solution) estimates obtained at $20{ }^{\circ} \mathrm{C}$. An additional file shows these estimates inside a dashed pointed rectangle (Additional file 4). The estimated time needed for 10-fold reduction in FMDV titres in spiked urine (lowest value) was 0.5 days, indicating an FMDV survival rate $(\sigma)$ of $0.014 \mathrm{day}^{-1}$. The estimated time needed for 10-fold reduction in FMDV titres in spiked faecal material (middle value) was 2.8 day indicating an FMDV survival rate $(\sigma)$ of 0.44 day $^{-1}$. The estimated time needed for 10 -fold reduction in FMDV titres in spiked buffered solution (highest value) was 8.2 days, indicating an FMDV survival rate $(\sigma)$ of $0.75 \mathrm{day}^{-1}$. For the quantification of FMDV transmission, we used the middle estimate i.e. $\sigma=0.44$ day $^{-1}$.

\section{Quantification of FMDV transmission Results of the 1R-SIR model}

The transmission rate parameter $\hat{\beta}$ was 0.67 per day $(95 \%$ CI: $0.26,1.8)$. The average infectious period from the inoculated calves $\hat{T}$ was 5.5 days (95\% CI: 4.5, 6.7). Therefore the estimated reproduction ratio $\hat{R}_{0}{ }^{1 \mathrm{R}}$ was 3.7 (95\% CI: 1.3, 10.), significantly above 1 .

\section{Results of the $2 R$ - SIR model}

The regression coefficient of $\mathrm{f}_{\mathrm{e}}$, the extra infectivity contributed by the environment, was not significantly different from 0 which means that $\beta_{\text {contact }}$ and $\beta_{\text {environment }}$ are not significantly different. Because $\beta_{\text {environment }} / \beta_{\text {contact }}$ equalled 1.4 (95\% CI 0.14, 14), there is contribution of the environment. Using the most parsimonious model $\beta_{\text {contact }}$ and $\beta_{\text {environment }}$ were estimated both to be 0.45 per day (95\% CI: $0.24,0.85)$. Because $\hat{T}$ was 5.5 days (95\% CI: 4.5, 6.7), $\hat{R}_{0}{ }^{2 R}$ contact equalled 2.5 (95\% CI: 1.3, 5.0). The average infectious period from the contaminated environment $\hat{\tau}$ was 4.3 days (95\% CI: 3.6, 5.2), which leads to a $\hat{R}_{0}{ }^{2 R}$ environment of 1.9 (95\% CI: 1.007, 3.8). Combination of the two estimates $\left(\hat{R}_{O}{ }^{2 R}\right.$ contact $+\hat{R}_{0}{ }^{2 R}$ environment $)$ resulted in $\hat{R}_{O}{ }^{2 R}$ equalled to 4.4 (95\% CI: $1.5,7.4)$, which is significantly above 1 . $\hat{R}^{2 R}$ was not significantly different from $\hat{R}_{0}{ }^{1 R}$ as can be seen from their overlapping confidence intervals. The contribution of the environmental transmission to the total transmission of FMDV was $44 \%$ $\left(\hat{R}_{0}{ }^{2 R}\right.$ environment $/ \hat{R}_{O}{ }^{2 R}$.) The sensitivity analysis, i.e. multiplication of the new infections or cases $(C)$ in $E_{t}$ by either 0 or 0.5 , resulted in the same contribution of the environmental transmission (44\%). When the lowest and the highest values of $\sigma$ were used, the contribution of the environmental transmission to the total transmission was estimated to be $31 \%$ (when $\sigma=0.014$ day $^{-1}$ ) and $75 \%$ (when $\sigma=0.75$ day $^{-1}$ ). The sensitivity analysis in which we included a latent period of 1, 2 or 3 days, resulted in higher estimates for $\beta$ (Additional file 5 ) and $R_{0}$
(Additional file 6) for the models with a latent period, but the estimated contribution of the environment stayed the same (Additional file 6).

\section{Results of the final size model}

The $\hat{R}_{O}{ }^{F S}$ equalled 14 (95\% CI: 1.3, infinite), which is significantly above 1 . Based on the comparison of the confidence intervals, $\hat{R}_{0}{ }^{F S}$ seems to be not significantly different from $\hat{R}_{0}{ }^{1 R}$ nor from $\hat{R}_{0}{ }^{2 R}$.

\section{Experiments with vaccinated calves}

After challenge, none of the inoculated or contact calves became infectious; therefore transmission parameters could not be estimated.

\section{Discussion}

In this study, we quantified the contribution of a contaminated environment to the transmission of FMDV and analysed whether vaccination one week prior to inoculation of the calves could block FMDV transmission. We show that using a 2R-SIR model allows FMDV transmission to be quantified in two parts: the direct contact component and the indirect i.e. via the environment component. Our results show that roughly $44 \%$ of the transmission of FMDV occurs via the environment, in the days after the calves started secreting and excreting the virus. The contribution of the environment to the transmission of FMDV depends on the FMDV survival rate; if the survival rate is high, the contribution of the environment is higher.

An environment that has previously housed infectious animals can contain FMDV if it is not properly disinfected after the removal of the infectious animals [5] and our study shows that this virus accumulation can cause new infections. As we show, environmental transmission of FMDV plays a role in the total transmission of FMDV also in groups of animals that do have direct contact. Transmission of FMDV has been quantified before in several studies by using a $1 \mathrm{R}$ SIR model [14,18,36-41]. We believe that in all of these studies, transmission occurred through both routes: via direct contact to an infected animal and via indirect contact to a contaminated environment. However within the experimental design of those studies, the role of the environment could not be separated from the role of direct contact on the transmission of FMDV. By using both direct and indirect contact experiments we could employ a 2R-SIR model (that included accumulation of FMDV in the environment) to quantify the contribution of the environment $\left(\hat{R}_{0}{ }^{2 R}\right.$ environment $)$ to the total transmission of FMDV. As expected, the estimated $\hat{R}_{0}{ }^{1 R}, \hat{R}_{0}{ }^{2 R}$ and $\hat{R}_{0}{ }^{F S}$ are very similar to each other and moreover, they are similar to the $\hat{R}_{0}$ (by using a 1R-SIR model) estimated in other 
direct contact experiments with cattle infected with FMDV O/NET/2001 [18,41]. The consistency of these results indicates that our 2R-SIR model is valid for the estimation of the reproduction ratio and that it is very useful to separate both components i.e. the environment and direct contact transmission, for the quantification of their separate contribution to the transmission of FMDV. Moreover based on the statistical analysis of virus secretion and excretion, the results obtained with the 2R-SIR model are not biased by the route of infection i.e. inoculated and contact infected calves.

In our models, we used an SIR model and we did not incorporate a latent period (then we would have a SEIR i.e. susceptible, exposed, infectious, recovered model), although the data from the virus excretion of the inoculated animals suggest that for this group there is a latent period of approximately 2 days. The main reason why we did not incorporate a latent period in our study is because we did not want to introduce more complexity in the model. Also, incorporation of a latent period affects the estimates for the direct and indirect transmission more or less equally and thus the estimation of the role of the environment (the main interest of this research) was not be affected. Our sensitivity analysis showed that, when a latent period is incorporated in the models, the estimates of the transmission parameters are still "equal" i.e. not significantly different (Additional files 5 and 6). The transmission parameters we provide in Additional files 5 and 6 , where a latent period was used, could be useful when the transmission parameters are applied for modelling disease outbreaks and the effect of control measures.

The temporal separation used in our indirect contact experiment allowed us to observe the occurrence of transmission through the environment by taking into consideration virus accumulation in 2 different periods i.e. $0-3$ and 3-6 dpi. Temporal separation was also used by Charleston et al. [42] to study FMDV transmission, although they exposed "donor" calves to "recipient" calves by direct contact for 8 hours in separate environments that had been previously disinfected, and thus with no accumulation of virus in the environment. This would, based on our results, reduce transmission of FMDV. They conclude in their study that the occurrence of FMDV transmission is correlated with the presence of clinical signs. However, it has been previously shown that FMDV transmission also can occur before clinical signs are seen [39]. In our study as well, transmission through the environment was caused by one group of calves that contaminated the environment from 0 to $3 \mathrm{dpi}$ but showed no clinical disease. This supports the conclusion that the correlation of FMDV transmission with the presence of clinical signs cannot be generalised to populations, if animals have direct contact to each other for a longer period and/or are present where accumulation of FMDV in the environment is plausible. FMDV transmission may not occur, however, when animals are separated by fences or wooden walls (in pigs [43]; in calves: Charleston et al. (personal communication), [20]), indicating that either exposure to virus secreting and/or excreting animals or exposure to virus contaminated surfaces is important for the occurrence of transmission.

Vaccination can be used as a tool to reduce transmission of FMDV [17]. In our study the calves vaccinated one week prior to inoculation with FMDV did not shed virus. Previously, vaccinating animals 2 weeks prior inoculation with FMDV was reported [18] to reduce FMDV transmission; our results indicate that vaccination reduces FMDV transmission even earlier. As others have demonstrated, vaccination rapidly protects cattle from clinical disease, and reduces virus shedding by infected cattle [44-46]. As our results indicate, vaccination as early as one week before challenge cannot only protect calves against infection but also, can avoid contamination of the environment and so prevent new infections.

In summary, our study shows that the environment is a relevant mechanism in the transmission of FMDV. The quantification of the magnitude of the contribution of transmission via the environment emphasises again that hygiene is an extremely important control measure for FMDV. And that, as already recommended by veterinary authorities, good disinfection of e.g. vehicles, walls and floors previously contaminated by infected animals is necessary to reduce the accumulation of the virus in the environment and therefore FMDV transmission. Also, the data from our experiment give some insight in which secretions and excretions contain FMDV at different times post infection and also this knowledge could be to improve control measures. The accumulation of FMDV in the environment should be taken into account when studying FMDV transmission. Further, the environmental aspect in the transmission of FMDV should be considered during the planning and implementation of measures to control FMD during an outbreak.

\section{Additional files}

Additional file 1: On the FMDV survival rate $\sigma$. Detailed calculation of the FMDV survival rate $\sigma$, which was calculated using published data on FMDV thermal inactivation combined with own laboratory data [7,20,23-27].

Additional file 2: The 2R-SIR model. Detailed information on the quantification of transmission rate parameters. The transmission rate parameters were calculated using a Generalized Linear Model (GLM) based on an stochastic SIR model. In this additional file we describe the SIR model parameters, the inclusion of an extra route i.e. E to the 1 route SIR-model to calculate the contribution of the environment to the transmission of the infection and, the methodology to quantify the transmission parameters using the GLM model [28,29].

Additional file 3: Mean values (plus range) and the Kruskal-Wallis statistics of virus present in secretions, excretions and blood samples, for the inoculated, direct contact and indirect contact groups. For the Kruskal-Wallis statistics, $\mathrm{H}$ is the Kruskal-Wallis test statistic and df the degrees of freedom. 
Additional file 4: Plotted linear regression estimates of the log time (hours) needed for a 10-fold reduction in FMDV titres. In this additional file we show the obtained times (log hours) that are needed to have a 10-fold reduction in FMDV titres per sample and per temperature. Light blue points correspond to estimates from water; green from buffers; grey from hemal and lymph nodes and bone marrow; black from faeces; red from urine; pink from milk; blue from slurry. Inside the dashed pointed rectangles, only obtained estimates at $20^{\circ} \mathrm{C}$. Red dashed lines, regression lines at $95 \% \mathrm{Cl}$.

Additional file 5: Sensitivity analysis considering latent periods. In this additional file we show results of the estimation of transmission parameters for latent periods of 0 (as used in the paper), 1, 2 and 3 days.

Additional file 6: Sensitivity analysis considering latent periods. In this additional file we show results of the estimation of $R_{0}$ 's for latent periods of 0 (as used in the paper), 1, 2 and 3 days.

\section{Competing interests}

The authors declare that they have no competing interests.

\section{Authors' contributions}

CB participated in the design and coordination of the study, participated in the laboratory analysis, carried out the statistical analysis and drafted the manuscript. MJ participated in the design of the study, carried out the statistical analysis and drafted the manuscript. PE participated in the design and coordination of the study and helped to draft the manuscript. AD conceived the study, participated in the design and coordination of the study, carried out the statistical analysis and helped to draft the manuscript. All authors read and approved the final manuscript.

\section{Acknowledgements}

The authors would like to thank Mrs. F. van Hemert-Kluitenberg for her assistance with the handling of samples and laboratory assays. The research leading to these results have received funding from the European Community's Seventh Framework Programme (FP7/2007-2013) under grant agreement n 226556 (FMD-DISCONVAC)

\section{Author details}

${ }^{1}$ Central Veterinary Institute (CVI), part of Wageningen UR, P.O. Box 65, 8200 AB Lelystad, The Netherlands. ${ }^{2}$ Department Quantitative Veterinary Epidemiology, Wageningen University, P.O. Box 338, 6700 AH Wageningen, The Netherlands.

Received: 6 December 2013 Accepted: 30 January 2015 Published online: 17 April 2015

\section{References}

1. Thomson GR (1994) Foot and Mouth Disease. In: Coetzer JAW, Thomson GR, Tustin RC (ed) Infectious diseases of livestock with special reference to Southern Africa. Volume 1. Oxford University Press, Capetown, pp 825-852

2. Woolhouse M, Chase-Topping M, Haydon D, Friar J, Matthews L, Hughes G Shaw D, Wilesmith J, Donaldson A, Cornell S, Keeling M, Grenfell B (2001) Epidemiology. Foot-and-mouth disease under control in the UK. Nature 411:258-259

3. Bravo de Rueda C, Dekker A, Eblé PL, de Jong MCM (2014) Identification of factors associated with increased excretion of Foot-and-Mouth Disease Virus. Prev Vet Med 113:23-33

4. Parker J (1971) Presence and inactivation of foot-and-mouth disease virus in animal faeces. Vet Rec 88:659-662

5. Sellers RF, Herniman KA, Donaldson Al (1971) The effects of killing or removal of animals affected with foot-and-mouth disease on the amounts of airborne virus present in looseboxes. Br Vet J 127:358-365

6. Botner A, Belsham GJ (2012) Virus survival in slurry: analysis of the stability of foot-and-mouth disease, classical swine fever, bovine viral diarrhoea and swine influenza viruses. Vet Microbiol 157:41-49

7. Turner C, Williams SM, Cumby TR (2000) The inactivation of foot and mouth disease, Aujeszky's disease and classical swine fever viruses in pig slurry. J Appl Microbiol 89:760-767

8. van Bunnik BA, Hagenaars TJ, Bolder NM, Nodelijk G, de Jong MCM (2012) Interaction effects between sender and receiver processes in indirect transmission of Campylobacter jejuni between broilers. BMC Vet Res 8:123
9. van Bunnik BA, Katsma WE, Wagenaar JA, Jacobs-Reitsma WF, de Jong MCM (2012) Acidification of drinking water inhibits indirect transmission, but not direct transmission of Campylobacter between broilers. Prev Vet Med 105:315-319

10. Breban R, Drake JM, Stallknecht DE, Rohani P (2009) The role of environmental transmission in recurrent avian influenza epidemics. PLoS Comput Biol 5:e1000346

11. Rohani P, Breban R, Stallknecht DE, Drake JM (2009) Environmental transmission of low pathogenicity avian influenza viruses and its implications for pathogen invasion. Proc Natl Acad Sci U S A 106:10365-10369

12. Velkers FC, Bouma A, Stegeman JA, de Jong MCM (2012) Oocyst output and transmission rates during successive infections with Eimeria acervulina in experimental broiler flocks. Vet Parasitol 187:63-71

13. Eblé $P L$, de Koeijer $A A$, de Jong MCM, Engel B, Dekker A (2008) A meta-analysis quantifying transmission parameters of FMDV strain O Taiwan among non-vaccinated and vaccinated pigs. Prev Vet Med 83:98-106

14. Orsel K, Dekker A, Bouma A, Stegeman JA, de Jong MCM (2007) Quantification of foot and mouth disease virus excretion and transmission within groups of lambs with and without vaccination. Vaccine 25:2673-2679

15. Kermack WO, McKendrick AG (1927) A Contribution to the mathematical theory of epidemics. Proc Roy Soc Lond 115:700-721

16. Velthuis AGJ, de Jong MCM, De Bree J, Nodelijk G, Van Boven M (2002) Quantification of transmission in one-to-one experiments. Epidemiol Infect 128:193-204

17. Cox SJ, Barnett PV (2009) Experimental evaluation of foot-and-mouth disease vaccines for emergency use in ruminants and pigs: a review. Vet Res 40:13

18. Orsel K, Dekker A, Bouma A, Stegeman JA, de Jong MCM (2005) Vaccination against foot and mouth disease reduces virus transmission in groups of calves. Vaccine 23:4887-4894

19. Hajer R, Hendrikse J, Rutgers LJE, van Oldruitenborgh-Oosterbaan MM S, van der Weyden GC (1985) Het klinisch onderzoek bij grote huisdieren. Wetenschappelijke uitgeverij Bunge, Utrecht

20. Bouma A, Dekker A, de Jong MCM (2004) No foot-and-mouth disease virus transmission between individually housed calves. Vet Microbiol 98:29-36

21. Moonen P, Boonstra J, van der Honing RH, Leendertse CB, Jacobs L, Dekker A (2003) Validation of a LightCycler-based reverse transcription polymerase chain reaction for the detection of foot-and-mouth disease virus. J Virol Methods 113:35-41

22. Dekker A, Terpstra C (1996) Prevalence of foot-and-mouth disease antibodies in dairy herds in The Netherlands, four years after vaccination. Res Vet Sci 61:89-91

23. Dekker A (1998) Inactivation of foot-and-mouth disease virus by heat, formaldehyde, ethylene oxide and gamma radiation. Vet Rec 143:168-169

24. Bachrach HL, Breese SS, Callis JJ, Hess WR, Patty RE (1957) Inactivation of foot-and-mouth disease virus by $\mathrm{pH}$ and temperature changes and by formaldehyde. Proc Soc Exp Biol Med 95:147-152

25. Blackwell JH, Hyde JL (1976) Effect of heat on foot-and-mouth disease virus (FMDV) in the components of milk from FMDV-infected cows. J Hyg 77:77-83

26. Hyde JL, Blackwell JH, Callis JJ (1975) Effect of pasteurization and evaporation on foot-and-mouth disease virus in whole milk from infected cows. Can J Comp Med 39:305-309

27. Cottral GE (1969) Persistence of foot-and-mouth disease virus in animals, their products and the environment. Bull Off Int Epizoot 70:549-568

28. Velthuis AG, de Jong MCM, Kamp EM, Stockhofe N, Verheijden JH (2003) Design and analysis of an Actinobacillus pleuropneumoniae transmission experiment. Prev Vet Med 60:53-68

29. Bouma A, de Jong MCM, Kimman TG (1995) Transmission of pseudorabies virus within pig-populations is independent of the size of the population. Prev Vet Med 23:163-172

30. McCullagh P, Nelder JA (1989) Generalized Linear Models. Boca Raton, Chapman \& Hall/CRC

31. R Development Core Team (2012) R: A Language and Environment for Statistical Computing. The R Foundation for Statistical Computing, Vienna

32. Diekmann O, Heesterbeek JA, Metz JA (1990) On the definition and the computation of the basic reproduction ratio $R_{0}$ in models for infectious diseases in heterogeneous populations. J Math Biol 28:365-382

33. de Jong MCM, Kimman TG (1994) Experimental quantification of vaccineinduced reduction in virus transmission. Vaccine 8:761-766

34. Velthuis AG, de Jong MCM, Stockhofe N, Vermeulen TM, Kamp EM (2002) Transmission of Actinobacillus pleuropneumoniae in pigs is characterized by variation in infectivity. Epidemiol Infect 129:203-214

35. Velthuis AG, de Jong MCM, De Bree J (2007) Comparing methods to quantify experimental transmission of infectious agents. Math Biosci 210:157-176 
36. Eblé PL, de Koeijer A, Bouma A, Stegeman A, Dekker A (2006) Quantification of within- and between-pen transmission of Foot-and-Mouth disease virus in pigs. Vet Res 37:647-654

37. Eblé $P L$, Bouma A, de Bruin MG, van Hemert-Kluitenberg F, van Oirschot JT, Dekker A (2004) Vaccination of pigs two weeks before infection significantly reduces transmission of foot-and-mouth disease virus. Vaccine 22:1372-1378

38. Goris NE, Eblé PL, de Jong MCM, De Clercq K (2009) Quantification of footand-mouth disease virus transmission rates using published data. Altex 26:52-54

39. Orsel K, Bouma A, Dekker A, Stegeman JA, de Jong MCM (2009) Foot and mouth disease virus transmission during the incubation period of the disease in piglets, lambs, calves, and dairy cows. Prev Vet Med 88:158-163

40. Orsel K, de Jong MCM, Bouma A, Stegeman JA, Dekker A (2007) Foot and mouth disease virus transmission among vaccinated pigs after exposure to virus shedding pigs. Vaccine 25:6381-6391

41. Orsel K, de Jong MCM, Bouma A, Stegeman JA, Dekker A (2007) The effect of vaccination on foot and mouth disease virus transmission among dairy cows. Vaccine 25:327-335

42. Charleston B, Bankowski BM, Gubbins S, Chase-Topping ME, Schley D, Howey R, Barnett PV, Gibson D, Juleff ND, Woolhouse MEJ (2011) Relationship between clinical signs and transmission of an infectious disease and the implications for control. Science 332:726-729

43. van Roermund HJ, Eblé PL, de Jong MCM, Dekker A (2010) No betweenpen transmission of foot-and-mouth disease virus in vaccinated pigs. Vaccine 28:4452-4461

44. Golde WT, Pacheco JM, Duque H, Doel T, Penfold B, Ferman GS, Gregg DR, Rodriguez LL (2005) Vaccination against foot-and-mouth disease virus confers complete clinical protection in 7 days and partial protection in 4 days: Use in emergency outbreak response. Vaccine 23:5775-5782

45. Doel TR, Williams L, Barnett PV (1994) Emergency vaccination against footand-mouth disease: rate of development of immunity and its implications for the carrier state. Vaccine 12:592-600

46. Cox SJ, Voyce C, Parida S, Reid SM, Hamblin PA, Paton DJ, Barnett PV (2005) Protection against direct-contact challenge following emergency FMD vaccination of cattle and the effect on virus excretion from the oropharynx. Vaccine 23:1106-1113

\section{Submit your next manuscript to BioMed Central and take full advantage of:}

- Convenient online submission

- Thorough peer review

- No space constraints or color figure charges

- Immediate publication on acceptance

- Inclusion in PubMed, CAS, Scopus and Google Scholar

- Research which is freely available for redistribution

Submit your manuscript at www.biomedcentral.com/submit 